\title{
La atención a la diversidad de los menores en conflicto con la ley Penal desde una visión integradora
}

\section{Attention to the diversity of minors in conflict with criminal law from an integrative perspective}

\author{
Aldo Chaviano Jiménez \\ Universidad Central "Marta Abreu" de las Villas. Cuba \\ ORCID: https://orcid.org/0000-0002-4995-2964 \\ Karel Llopiz Guerra \\ Universidad Central "Marta Abreu" de las Villas. Cuba \\ ORCID: https://orcid.org/0000-0002-1500-8000 \\ Alexander Vladimirovich Miroshnichenko \\ Universidad Federal del Sur. Rostov del Don. Russia. \\ ORCID: https://orcid.org/0000-0003-0831-3127 \\ Mónica López-Narvaes \\ Universidad César Vallejo, Perú \\ ORCID: https://orcid.org/0000-0002-8399-5677 \\ Yolanda Chipana-Fernández \\ Universidad Cesar Vallejo, Perú \\ ORCID: https://orcid.org/0000-0002-8609-3409 \\ Gaby Nieto-Fernández \\ Universidad Cesar Vallejo, Perú \\ ORCID: https://orcid.org/0000-0003-0303-9915
}

*Correspondence

Email: aldoch@jnauta.cu
Cite as: 


\section{Resumen}

Responder a las necesidades del mejoramiento profesional y humano atendiendo a la diversidad, es una de las exigencias de los sistemas educativos. Estudios internacionales realizados por diversos autores han demostrado preocupaciones por la atención a los menores que se encuentran en conflicto con la Ley. El objetivo de este trabajo se refiere a un estudio de revisión por diferentes profesionales del orbe, acerca de la atención que reciben los menores que se encuentran en conflicto con la ley penal, así como las causas que lo originan, líneas de trabajo y estrategias a desarrollar para su prevención y atención en todos los niveles desde la intersectorialidad.

Palabras claves: Docentes, Menores, Niños, Problemas Sociales, Ley, Prevención.

\section{Summary}

Responding to the needs of professional and human improvement by attending to diversity is one of the demands of educational systems. International studies carried out by several authors have shown concerns about the attention to minors in conflict with the law. The objective of this work refers to a review study by different professionals of the world, about the attention received by minors in conflict with the criminal law, as well as the causes that originate it, lines of work and strategies to be developed for their prevention and attention at all levels from the intersectoriality.

Keywords: Teachers, children, social problem, low, prevention.

\section{Introducción}

A nivel mundial constituye una necesidad la atención a la diversidad de niños, adolescentes y jóvenes que se encuentran vinculados a los sistemas educativos y por determinadas razones se encuentran en conflicto con la Ley Penal. Principalmente cuando el resto del mundo se encuentra inmerso en tareas vinculadas con la atención educativa a la diversidad.

Responder a las necesidades del mejoramiento profesional y humano atendiendo a la diversidad, es una de las exigencias de los sistemas educativos en el mundo lo cual exige el perfeccionamiento de los propios sistemas, teniendo en cuenta que el desarrollo integral de un individuo, no es posible lograrlo sin la dedicación requerida y el empleo de métodos eficientes en la tarea de educación e inserción del menor en las actividades sociales y educativas.

Estudios internacionales realizados por diversos autores han demostrado preocupaciones por la atención a los menores que se encuentran en conflicto con la Ley, las que han tenido sus espacios en los Congresos de la ONU, UNICEF, donde se han tomado líneas de trabajo, para su atención en todos los niveles.

La expresión "niños y niñas en conflicto con la ley" se refiere a cualquier menor de 18 años que entre en contacto con el sistema judicial por ser sospechoso o estar acusado de cometer algún delito. UNICEF (2017)

Desde un punto de vista histórico, las preocupaciones con la defensa jurídica de los menores de edad son nuevas. La defensa jurídica fue siempre considerada un mal y ni siquiera 
necesario.

Como se ha podido apreciar en Cuba, lo establecido en las Naciones Unidas al respecto, se ha venido cumpliendo, se les brindaba una especial atención a los menores que se encontraban en conflicto con la Ley penal teniendo en cuenta las principales causas que lo llevaban a verse en esta situación.

\section{Desarrollo}

Algunas de las manifestaciones desviadas de la conducta -deambular, deserción escolar, bajo rendimiento académico, disfuncionalidad en la familia, dificultades para acatar reglas sociales y/o grupales, deficientes relaciones interpersonales con adultos y coetáneos, etc.- cuando permanecen estables y perdurables en el tiempo, traen consigo que se produzca aquella transgresión a las normas legales y de la sociedad. Vega (2017).

Notable ha sido el aumento de menores en conflicto con la Ley Penal en el resto mundo, por lo que cada país ha realizado un esfuerzo desmedido por adecuar sus formas de actuar contra los niños infractores a fin de buscar caminos justos y a tono con el derecho penal moderno. La atención a los menores que se encuentran en conflicto con la Ley, dándole a estos un tratamiento específico teniendo en cuenta las principales características como la pobreza, la marginalidad, la falta de equidad, el nepotismo, la presencia de padres divorciados o padres que presentan problemas de conductas antisociales, así como los modos de convivencias con personas en conflicto con La Ley Penal, entre otras causas que constituyen la creciente delincuencia en la juventud.

En ese sentido, es central e indispensable tener en cuenta, a la hora de sancionar legislación, implementar políticas públicas, tomar decisiones judiciales, administrativas y de toda índole, lo señalado por todos los instrumentos de protección de derechos humanos, y en particular por la Convención sobre los Derechos del Niño, en la integralidad de su articulado. UNICEF (2015).

Con la ratificación de la Convención sobre los Derechos del Niño y la vigencia de las normativas internacionales conexas referidas al resguardo de derechos humanos de los niños y adolescentes, rige el principio de no regresividad, en el sentido que esta normativa es parte de la normativa interna y por lo tanto, no es posible retroceder en relación a los avances normativos logrados para la exigibilidad de derechos. Así también, se debe tener en cuenta el principio de integración, que alude a la aplicación de todo el corpus juris que protege los derechos y garantías de los adolescentes en conflicto con la ley penal. Barletta (2015)

Los trastornos de la conducta son factores que afectan la esencia de la naturaleza humana del hombre como ser social, sus manifestaciones se expresan en el contexto social y son determinadas en parte importantes por este. Estos trastornos ocasionan serios problemas en la vida personal, familiar y social de los escolares que los presentan, pues provocan en ellos sentimientos de desesperanza y frustración, conductas inadecuadas a las condiciones ambientales que resultan molestas para los demás, tanto adultos como coetáneos, muchas veces ocasionan rechazo y violencia en las personas que los rodean, lo que los lleva a asumir la vida con una perspectiva negativa. 
Según autores como Sánchez (2016) afirma que en este orden aparece la necesidad social de que estos escolares cambien, modifiquen su conducta en el menor tiempo posible, y ello requiere perfeccionar el proceso de atención educativa en los centros educacionales cubanos. Hernández, Barrabi (2018). En tal sentido una de las prioridades del Ministerio de Educación es: profundizar en el estudio sobre la eficiencia de la atención a escolares con trastornos de la conducta.

Si se tiene en cuenta que en muchas ocasiones los escolares con trastornos de la conducta suelen atacar, agredir de diversas formas el medio ambiente, se impone la toma de medidas urgentes, para preservar la calidad de este y remediar en lo posible los daños causados, de modo que podamos legar a las futuras generaciones un planeta habitable. Estas son las razones que inevitablemente proponen un desafío ante los educadores que trabajan con los escolares con trastornos de la conducta a utilizar la Educación Ambiental como recurso psicopedagógico en la atención educativa a estos escolares (Hernández y Barrapi, 2015).

A juicio de Morales (2020) siendo el Perú un país en vías de desarrollo, es relativamente fácil predecir el destino de muchos adolescentes, especialmente de aquellos que provienen de familias de escasos recursos económicos. Las variables mediadoras del desarrollo como la familia, la escuela, los niveles nutricionales, y la calidad de las experiencias normativas resultan poco diferenciadoras al momento de explicar la dirección del desarrollo en la gran mayoría de los adolescentes pobres. Esta condición incrementa el nivel de vulnerabilidad endógena y el riesgo social, representando una potencial fuente de costos sociales futuros expresados en índices elevados de desempleo, analfabetismo, delincuencia, drogodependencia, prostitución, morbi-mortalidad física y mental, y reproducción generalizada de la pobreza.

Ante este referente vinculado con este tema a criterio de Llopiz (2018), aun cuando se realiza divulgación por los medios de difusión masiva, la prensa escrita y el trabajo comunitario de médicos y enfermeras de la familia acerca de los daños que para la salud ocasionan el mal hábito de fumar, el consumo desmedido de bebidas alcohólicas y drogas ilegales, es imprescindible la labor preventiva que deben desarrollar las instituciones escolares, y en especial los centros de educación superior, en los cuales es preciso la consolidación y sistematicidad de las acciones educativas que conduzcan a evitar estos hábitos indebidos en los educandos.

Según Silva (2015) la influencia del Derecho Romano en el pensamiento jurídico se hizo sentir con evidente énfasis en relación con los menores, pues entre otros aportes, se fijaron y tuvieron en cuenta, las distintas etapas de edades, a las que les correspondía una determinada medida, por ello se establecieron categorías que distinguieron los límites para las diferentes respuestas penales que se aplicaban a los niños, entre otras disposiciones que beneficiaron paulatinamente la adecuada protección jurídica de los niños que transgredían las normas.

Con el decursar del tiempo aparece una nueva concepción sobre los derechos del niño en un justo reconocimiento como seres humanos, este nuevo punto de vista, ha ido fortaleciéndose con la aprobación de varios Instrumentos Internacionales, entre ellos La Convención de los Derechos del Niño, así de la "Doctrina de La Situación Irregular", se avanza a la "Doctrina de La Protección Integral", la que a su vez se apoya en que "nada es más importante que el interés del niño"

Cuando nos referimos a los menores, partimos de una categoría socialmente construida y 
por lo tanto histórica: la infancia constituye el resultado de un complejo proceso de definiciones, acciones institucionales y cambios sociales en los sentimientos de los mismos, que no se realizan en contextos políticamente neutros. Gallardo (2017)

Nieto (2012) explica que la delincuencia juvenil refleja las circunstancias que van ocurriendo en cada sociedad junto con las particularidades que envuelven los actos delictivos de los menores, que han ido cambiando de una manera progresiva; antes eran menores en situación de precariedad familiar, económica y/o social, pero el perfil ha sido trasformado con el transcurso del tiempo. La situación en la que se puede ver envuelta una persona en relación a la insuficiencia de recursos, precariedad, exclusión, marginalidad, etc., no puede confundirse con la situación delincuencial, aunque sí es cierto, que en numerosas ocasiones conduce al conflicto con la ley.

Asimismo, en otro de los estudios de Nieto (2009) expone que, estas circunstancias se pueden desencadenar por factores o circunstancias personales o sociales que llevan a la persona a entrar en un proceso discordante con las normas de la sociedad en la que vive. Esta inadaptación del individuo ante la sociedad es susceptible de generar infracciones y producir conductas disruptivas y antisociales, ya sea por una deficiente socialización, por la influencia del contexto desfavorable sobre la persona o conductas generadas de forma más tardía causadas por el aprendizaje social. (Gallardo, 2017)

Por otra parte, uno de los aspectos que con más peso cuenta en las investigaciones sobre conducta delictiva juvenil es el consumo de tóxicos. Droga y conducta delictiva aparecen frecuentemente asociadas en diferentes investigaciones encontrándose un patrón consistente de abuso de diferentes sustancias en los/las menores infractores/as y con conductas antisociales (Botija y Aragón, 2011; Crespo, Perles y San Martín, 2006, Botija, Aguair y Climen, 2018).

La propia Doctrina de La Situación Irregular: surge a finales del Siglo XIX, como una necesidad de "separar" a los niños involucrados en conductas típicas del procedimiento que se seguía para con los adultos, en este momento se comenzó a ver al niño infractor de normas penales como inimputable por razón de la edad y desde un punto de vista tutelar, como un ser incompleto. Bajo la concepción de esta Doctrina, el niño es considerado como un objeto y no sujeto de derecho.

Esta Doctrina constituye toda la elaboración teórica surgida con posterioridad a la creación de los tribunales de Chicago en 1899 y que permitió el desarrollo de un derecho de menores que se diferenció del de los adultos tanto en lo penal como en el ámbito de la protección jurídica, además trataba de abandonar todo el rigor inflexible y dotar a los tribunales de facultades ilimitadas con el propósito del amparo jurídico y la reeducación de los niños.

Este derecho de menores en la práctica carecía del carácter protector de los derechos y garantía del niño ya que las disposiciones que lo amparaban eran por lo general tanto o más represiva que la de los adultos, irrespetando de esta forma en no pocas veces sus derechos y garantías constitucionales y tiene como característica predominante que no diferencia el ámbito tutelar del penal tratando por igual al menor abandonado y al que ha cometido una infracción de La Ley.

Según refiere García (2012), la Doctrina de la Situación Irregular "constituye en realidad una colcha de retazos del sentido común que el destino elevó a categoría jurídica. Su misión consiste en realidad, en legitimar la disponibilidad estatal absoluta de sujetos 
vulnerables, que precisamente por serlo son definidos en situación irregular. En este sentido las hipótesis de entrada en el sistema carecen de la menor taxatividad.

Por su parte la Doctrina de la Protección Integral: constituye una nueva concepción ideológica, filosófica, jurídica y social respecto a la infancia.

A partir de la entrada en vigor de la convención de los derechos del niño que se sustenta en esta doctrina, los niños no son considerados como objetos, sino como sujetos de derechos y se fortalece la responsabilidad de los gobiernos y los adultos respecto a ellos.

En esta doctrina el menor deja de ser considerado objeto de la compasión-represión para ser considerado dentro de la categoría infancia-adolescencia como sujeto pleno de derechos, y en materia penal considera los hechos cometidos por el menor como faltas o delitos sobre los que tiene responsabilidad.

Varios han sido los momentos por los que ha atravesado cuba en la atención a aquellos menores que se encontraban en conflicto con la ley, en los cuales se destacan:

$>$ Puesta en vigor del Código Español de 1870 que se hizo vigente en Cuba por Real Decreto de 23 de mayo de 1979, y constituye el primer cuerpo jurídico que regula los diferentes aspectos acerca de los menores transgresores, conceptuándolos como sujetos de Derecho Penal (establece la responsabilidad penal de los menores a los 9 años).

$>$ En 1883, se funda el asilo "El Buen Pastor", para recluir a niños de nueve a once años que eran acusados de prostitución, carácter violento y otros comportamientos irregulares.

> Posteriormente en 1900 a través de una orden militar se crean las Escuelas Correccionales de Guanajay y de Aldecoa (para varones y hembras respectivamente)

$>$ En 1936 se promulga el Código de Defensa Social (Decreto-Ley 802 de 4 de abril de 1936) que aunque mantenía a los menores dentro del Derecho Penal, establecía el límite de la exención de la responsabilidad penal a la edad de 12 años, y la atenuaba a los mayores de 12 años y menores de 18 años, lo que se consideraba como circunstancia de menor peligrosidad.

$>$ En 1938 se crea mediante la Ley del 23 de junio un Centro de Orientación Infantil. Donde se concebía al niño en dos vertientes: el niño en peligro y el niño peligroso de cuya distinción depende las medidas que se aplican.

> Existieron también Las Casas de Observación, creadas a partir de la Ley 548 de 15 de septiembre de 1959, que se ocuparon de la custodia provisional, observación y del diagnóstico de aquellos menores de 18 años que cometieran actos calificados como delitos $\mathrm{o}$ contravenciones.

$>$ El seis de junio de 1959 se crea el Ministerio del Interior (MININT) quien privilegió dentro de su trabajo la prevención de la delincuencia.

> En el año 1961, cuando se disuelve el ministerio de Bienestar Social, el Ministerio de Educación asume la responsabilidad de la custodia provisional de aquellos menores que incurrieran en conductas antisociales.

$>$ A partir de 1964, los menores transgresores comienzan a ser juzgados por una sala especial de la entonces Audiencia de la Habana

$>$ En 1966 se crea el primer Centro de Evaluación, Análisis y Orientación de Menores de la Habana (CEAOM) y en 1974, como parte de la estrategia de lograr un mayor acercamiento a la problemática de los menores en conflicto con la ley penal, personal especializado de estos centros comenzaron a realizar evaluaciones socio-psico-pedagógicas para que el resto de los Tribunales Provinciales y Regionales tuvieran elementos para imponer las medidas a los 
menores transgresores.

$>$ En 1975 fue elaborado el primer Proyecto de Código de Menores.

$>$ En 1979 se crea la Ley 21 (Código Penal) el cual estableció la responsabilidad penal a partir de los 16 años, pero dispone transitoriamente que para los menores de 14 años de edad hasta los 16 años se continúe aplicando lo establecido en el Código de Defensa Social. Hasta que se promulgara una ley relativa a los menores transgresores lo cual no se logró hasta 1982 que se crea el Sistema para la Atención a Menores con trastornos de conducta a través del Decreto-Ley $64 / 82$

Se ha desarrollado una red de instituciones especializadas en la reinserción social de menores (refugios sociales para niños y adolescentes, centros de rehabilitación social y centros de asistencia para menores desamparados) a fin de darles rápidamente refugio hasta que se les ofrezca una solución definitiva para alejarlos de entornos familiares adversos que amenacen su vida y su desarrollo, así como para ejercer una labor de reinserción social con las familias y los niños. ONU (2012)

Según el Instituto Interamericano del Niño, un sistema de protección integral es básicamente un diseño organizacional y operativo concebido para la implementación de las políticas públicas de infancia y adolescencia, que tiene como paradigma la Convención de los Derechos del Niño. Este sistema trata de estructurar y sistematizar la relación entre todos los actores a los efectos de dar efectividad a los derechos reconocidos en la expresada Convención.

En Cuba, se ha investigado por diferentes profesionales las conductas de los menores, así como las causas que lo originan, entre ellos se destacan, Fleites (2003); García, (2005); Molina (2005) y Rivero (2005). Los cuales han desarrollan temáticas donde se implica la labor o trabajo de la Escuela de Formación Integral a partir de los resultados en determinados aspectos de la atención y tratamiento a dichos alumnos; siendo aún insuficiente la preparación de los docentes en la determinación y atención a los menores que se encuentran en conflicto con la ley.

Cuba al igual que el resto del mundo establece un cuerpo legislativo para la atención a los menores que se encuentran en conflicto con la ley penal, el cual se encuentra vigente desde 1882 , donde se establecen las categorías que determinan la presencia de un menor en conflicto con la ley, por lo que se hace necesario el conocimiento legislativo por parte de los docentes de las diferentes enseñanzas educativa en cuba, para de esta forma poderles dar el seguimiento adecuado y así lograr su reinserción o reeducación.

El cuerpo legislativo cubano para la atención a menores en conflicto con la Ley, establece tres categorías que llevan al niño a ser "sujetos" del mismo:

1- aquellos niños que presentan indisciplinas graves o trastornos permanentes de la conducta, que por su "desajuste" se les dificulta el aprendizaje en el Sistema Nacional de Educación;

2- aquellos cuya conducta sea disocial o manifestaciones antisociales que no lleguen a constituir índices significativos de peligrosidad social, tales como daños intencionales o imprudentes, algunas apropiaciones de objetos, lesiones que no tengan mayor entidad y escándalo público entre otras conductas poco peligrosas de acuerdo con el alcance de sus consecuencias, (ya en esta categoría se incluyen acciones típicas de delito) y

3- y aquellos que incurran en hechos de "elevada peligrosidad social" donde aparecen conductas típicas de delito o conductas antisociales que evidencien índices significativos de desviación o peligro social. 
El término menor de edad en Cuba establecido en el Código Civil de la república aprobado por la Asamblea Nacional del poder Popular el 16 de julio de 1987, establece en su artículo 29 la distinción entre mayores y menores de edad, estableciendo que se adquiere la mayoría de edad al alcanzar los 18 años cumplidos o por el matrimonio. La legislación penal cubana establece además que solo son imputables los mayores de 16 años y reconoce un tratamiento diferenciado al comisor de un hecho delictivo que se encuentre entre las edades de 18 y 20 años pudiéndose realizar adecuaciones a las sanciones en sus límites mínimos y máximos.

El esquema cubano referido al tema es original y como bien se señala en las categorías antes mencionadas no solo incluye a los menores en conflicto con la ley panal sino también a aquellos que presentan indisciplinas graves, trastornos permanentes de la conducta o manifestaciones antisociales que no lleguen a constituir índices significativos de desviación o peligrosidad social, teniendo como objetivo principal la reorientación o reeducación de los menores regido conjuntamente por el MINED y el MININT quienes a su vez se reparten la competencia de acuerdo a las categorías en que se encuentre el menor.

Es en este punto donde juega un papel fundamental la preparación del docente para la atención a estos menores ya que los mismos se pueden encontrar en las diferentes etapas del proceso de enseñanza.

La necesidad de formar pedagógicamente a los docentes para que puedan enfrentar los nuevos retos de la Educación en cuba, precisa facilitarles la adquisición de conocimientos relevantes, lo cual es un requerimiento de la práctica educativa y de la formación profesional que requiere el Sistema Educativo, para formar profesionales altamente competentes, capaces de brindar atención educativa a la diversidad.

Las bases teóricas que sustentan la superación profesional del docente han sido tratadas con anterioridad por varios autores cubanos como Berges (2003), Bernaza (2004), Fraga (2005); López et al. (2014), Morales (2010), Perdomo (2010) los que, en sentido general, sostienen que es un proceso continuo y permanente que se desarrolla a lo largo de la vida profesional del docente en ejercicio, elevando la competencia y el desempeño profesional siempre que responda a las propias necesidades del docente en el contexto de la actividad fundamental que realiza, a las del sistema educativo, y sus proyectos de vida. (Torres-Alfonso, 2018)

En las actuales condiciones de perfeccionamiento, donde los grandes desafíos imponen a los seres humanos la necesidad de ser cada día más eficaces y eficientes en su desempeño, se ha introducido con fuerza cada vez mayor el término competencia en el contexto de las diferentes profesiones y perfiles ocupacionales existentes. Siendo así los autores de este trabajo se afilian a la idea de que la competencia es la capacidad de reunir un conjunto de recursos para resolver con eficacia situaciones complejas, que puedan surgir en el ámbito laboral.

Dentro del perfeccionamiento de la Educación en Cuba uno de los objetivos centrales ha estado en la formación del maestro por su innegable influencia en la educación de niños y jóvenes y en lograr que la escuela sea el centro más importante de la comunidad, donde se produce la acción de los diferentes gestores educativos, que velan por atenuar los efectos sociales ajenos al fin de la educación y promover las condiciones educativas más favorables para el pleno desarrollo de cada individuo en su contexto y realidades.

En el caso específico de los docentes, los autores de este trabajo nos afiliamos además a la idea de que las competencias del profesional de la educación son aquellas que permiten 
solucionar los problemas inherentes al proceso pedagógico en general y al proceso de enseñanza aprendizaje en particular en el contexto de la comunidad educativa escolar y en correspondencia con el modelo del profesional de la educación, con el propósito de promover el desarrollo integral de la personalidad de los estudiantes, teniendo en cuenta los diversos factores que puedan aparecer en la práctica educativa.

En este sentido y con relación a la atención educativa a la diversidad sobre el menor que se encuentra en conflicto con la Ley Penal, son precisas las acciones que debe conocer el docente para su atención, las que se encuentran establecidas en el propio cuerpo legislativo para la Atención a menores que se encuentran en conflicto con la Ley Decreto ley 64 de 1982, en las cuales se determinan diferentes acciones a tener en cuenta las cuales son de carácter obligatorio por parte del menor, las mismas está establecidas de acuerdo a las categorías en que se encuentre y son controladas por las autoridades competentes.

Entre las distintas medidas del sistema nacional de atención a menores con trastornos de conductas se encuentran:

Medidas de internamiento o asistencia obligatoria a una escuela de conducta del Ministerio de Educación o Internamiento en una Escuela de Formación Integral del Ministerio del Interior.

Internamiento obligatorio en un establecimiento asistencial de la red de Centros del Ministerio de Salud Pública.

$>$ Obligación de tratamiento médico ambulatorio.

Vigilancia y Atención por el Ministerio del Interior.

> Vigilancia reforzada de los padres, tutores o de los que tengan a su cargo al Menor.

$>$ Atención individualizada en las propias Escuelas del Ministerio de Educación sin necesidad de internamiento.

Ubicación del menor como aprendiz de oficio en una unidad laboral, y de acuerdo con lo establecido con la legislación laboral vigente.

Atención por los Trabajadores Sociales de La Federación de las Mujeres Cubanas.

Dichas medidas implican la evaluación periódica del Menor, con el fin de que los Consejos Provinciales de Atención a Menores, determinen la sustitución de las medidas de internamiento por medidas de carácter externo o la cesación de estas. Siendo importante señalar que cualquier oposición de los padres, tutores u otras personas que tengan bajo su cuidado a menores, al cumplimiento de una medida u otra disposición de los Órganos del Sistema, constituirá un delito de Otros Actos Contrarios al Normal Desarrollo del Menor, que se materializará a partir de poner dichos representantes legales a disposición del Fiscal, como representante universal de los menores y quien según lo establecido en el Decreto-Ley 64/82 tiene las facultades siguientes:

Facultades del Fiscal en el esquema jurídico:

Realizar visitas de control de la legalidad a las unidades de la PNR para verificar el cumplimiento de lo establecido en la atención a menores que hayan incurrido en conductas infractoras o hechos tipificados como delitos.

> Visitar las escuelas de conducta y centros de reeducación de menores para comprobar el cumplimiento de las normas establecidas para la permanencia y tratamiento de los menores que se encuentren en esos centros. 
Examinar todo tipo de documentación relativa a la situación de los menores, así como efectuar entrevistas a éstos, a los maestros, psicólogos, pedagogos, trabajadores sociales, juristas y otros funcionarios encargados de la educación y reorientación de los menores.

Diversos son los órganos que ejecutarán lo preceptuado en dicho cuerpo legislativo, regidos por los ministerios de educación y del interior, quienes tienen la tarea de dar a los menores un seguimiento que les permita ir evaluando las conductas y acciones de estos.

Los Consejos Provinciales de atención a menores: disponen las medidas que correspondan a los menores enmarcados en las categorías antes señaladas, vigilan su ejecución y deciden cualquier cambio en las medidas dispuestas. Estos Consejos Provinciales deciden la permanencia del niño en la Escuela de Formación Integral, hasta los 18 años si al cumplir 16 años la reeducación no se ha completado; el niño que participe en hechos intencionales tipificados en el Código Penal y represente "alta peligrosidad", al llegar a los 18 años de edad, el Consejo Provincial podrá decidir internarlo en un Centro de Mayores (prisión) por un término que no exceda de 5 años, el término del internamiento de los niños estará en función de los progresos que el mismo demuestre durante su cumplimiento, lo que será valorado por los especialistas del Centro donde se encuentre, los propios Consejos Provinciales determinarán la cesación o sustitución de las medidas de internamiento.

La decisión de cada uno de estos Consejos conlleva previamente un proceso evaluativo a cargo de los Centros de Diagnóstico y Orientación del MINED y los Centros de Evaluación Análisis y Orientación de Menores (CEAOM) del MININT quienes investigan y analizan las condiciones familiares y sociales de los menores

Los Centros de Diagnóstico, recomiendan la medida a adoptar:

Las Escuelas de Conducta, tratan de lograr que los alumnos se integren a la vida escolar y social.

Las Escuelas de Formación Integral, tienen como objeto modificar los malos hábitos, incidir en la formación y lograr modificar su conducta para integrarlo a la vida escolar, laboral y social, mientras que la policía se encarga de investigar los hechos en que hayan participado los niños incluyendo su conducta en general.

Las misiones de estos órganos que intervienen en dicho sistema se encuentran en la prevención, reeducación y reinserción a la sociedad de los menores enmarcados en las categorías establecidas por este cuerpo legislativo (D. Ley 64/82).

Teniendo en cuenta todo lo antes expuesto acerca de la atención al menor que se encuentra en conflicto con la Ley penal, el autor se acoge al criterio de fortalecer en los docentes sus conocimientos legislativos acerca del tema para de esta forma poder contribuir a la atención, de estos menores desde el punto de vista de la diversidad.

\section{Conclusiones}

La atención educativa a la diversidad se ha ido fortaleciendo al mismo tiempo que el reto del mundo se esfuerza por mejorar las formas de actuación con todos aquellos que de una forma $u$ otra forman parte de la diversidad tanto en el sector educativo como en la sociedad en general, donde se encuentran no solo aquellos estudiantes con algún tipo de discapacidad intelectual, sino 
que unido a estas se pueden presentar las indisciplinas graves o trastornos permanentes de la conducta, lo cual los convierte en sujetos del cuerpo legislativo para la atención amenores en conflicto con la ley por estar enmarcados en la primera categoría establecido por los Decreto Leyes referidos a la protección y atención a estos menores. Por lo que es imprescindible y necesario que el personal docente tenga dominio del mismo y así pueda brindar una atención adecuada y que satisfaga las demandas en la construcción de sociedades justas y donde prevalezca el orden y la atención a estos infantes.

\section{Referencias}

Barletta, V. M. (2015). LA JUSTICIA PENAL JUVENIL EN EL PERÚ: Un aporte para la construcción de un sistema penal garantista y de reinserción socio familiar. Recuperado de: https://bice.org/app/uploads/2015/12/40.pdf

Botija, M. Aguiar, F.F. Cilment, M. L. (2018). Adolescencias y riesgos: escenarios para la socialización en las sociedades globales. Revista Prisma Social, 23.

Chaviano, J. A. (2015). Los menores en conflicto con la Ley Penal, Enfoque Cubano. Los menores en conflicto con la Ley Penal, Enfoque Cubano. Villa Clara, Cuba.

Gallardo, C. C. (2017). Características socioeducativas de los menores en conflicto con la Ley. Universidad de la Laguna, Facultad de Ciencias Políticas, Sociales y de la Comunicación.

García, M.E. (2009). Los menores de edad en conflicto con la ley y el problema de los criterios de eficacia de la defensa jurídica: un nudo gordiano. Revista Isonomía, 31. Recuperado de http://www.scielo.org.mx/scielo.php?script=sci_arttext\&pid=S140502182009000200006

García, M. E. (2012). De las relaciones públicas al neomenorismo: 20 años de convención internacional de los derechos del niño en america latina (1989-2009) Passagens. Revista Internacional de História Política e Cultura Jurídica, 3(1), 117- 141. doi: 10.5533/19842503-20113106

Hernández, P. S. y Barrabi, M. M. (2018). La Atención Educativa Integral a los escolares con trastornos de conducta. Pinar del Río. Recuperado de: http://rc.upr.edu.cu/jspui/handle/DICT/3020

Llopiz, G. K. (2018). Educación antitabáquica en escolares primarios desde la labor extensionista universitaria. Revista Edumecentro, 10(3). Recuperado de http://www.revedumecentro.sld.cu/index.php/edumc/article/view/1197/pdf_335

Morales, C. H. (2020). El Adolescente infractor en conflicto con la Ley Penal: Una perspectiva sociopsicológica del Sistema de Justicia Penal Juvenil en el Perú. Observatorio Internacional de justicia Juvenil. Recuperado de: https://www.oijj.org/centrodocumental/docs/general/el-adolescente-infractor-en-conflicto-con-la-ley-penaluna

Nieto, M. C. (2012). La delincuencia juvenil. Libros y Revistas Intervención con menores en conflicto con la ley. Recuperado de: https://libros-revistasderecho.vlex.es/vid/delincuencia-juvenil-646065921 
ONU. (2012). Justicia en asuntos concernientes a los niños en conflicto con la ley. Informe a la ONU.

Silva, F. d. (2015). La protección jurídica de los niños en conflicto con la ley. la protección jurídica de los niños en conflicto con la ley. La Habana, La Habana.

Torres-Alfonso, A. M. (2018). Preparación del docente para perfeccionar su competencia didáctica en las escuelas superiores pedagógicas angolanas. EduSol, 13.

UNICEF. (2015). Relevamiento nacional sobre adolescentes en conflicto con la ley penal. año 2015.

Vega. A. (2017). La doble victimización de adolescentes en conflicto con la norma legal. Revista Universidad de la Hbna, 283. Recuperado de http://scielo.sld.cu/scielo.php?script=sci_arttext\&pid=S0253-92762017000100009 